\title{
Facile Synthesis of Micron-Sized Hollow Silver Spheres as Substrates for Surface-Enhanced Raman Scattering
}

\author{
Lixin Xia, ${ }^{1}$ Jiarui Xia, ${ }^{1,2}$ Ran Wei, ${ }^{1}$ Ying Sui, ${ }^{1}$ Zhipeng Sun, ${ }^{1}$ Xuehua Song, \\ Peng Song, ${ }^{3,4}$ and Lini Yang ${ }^{1}$ \\ ${ }^{1}$ College of Chemistry, Liaoning University, Shenyang 110036, China \\ ${ }^{2}$ School of Chemistry and Chemical Engineering, Shandong University, Jinan 250100, China \\ ${ }^{3}$ College of Physics, Liaoning University, Shenyang 110036, China \\ ${ }^{4}$ Liaoning Key Laboratory of Semiconductor Light Emitting and Photocatalytic Materials, Liaoning University, Shenyang 110036, China
}

Correspondence should be addressed to Lixin Xia; lixinxia@lnu.edu.cn and Lini Yang; lnyangg@gmail.com

Received 18 March 2014; Accepted 21 September 2014; Published 19 October 2014

Academic Editor: Mark van Der Auweraer

Copyright (c) 2014 Lixin Xia et al. This is an open access article distributed under the Creative Commons Attribution License, which permits unrestricted use, distribution, and reproduction in any medium, provided the original work is properly cited.

\begin{abstract}
A well-designed type of micron-sized hollow silver sphere was successfully synthesized by a simple hard-template method to be used as substrates for surface-enhanced Raman scattering. $4 \AA$ molecular sieves were employed as a removable solid template. $\left[\mathrm{Ag}\left(\mathrm{NH}_{3}\right)_{2}\right]^{+}$was absorbed as the precursor on the surface of the molecular sieve. Formaldehyde was selected as a reducing agent to reduce $\left[\mathrm{Ag}\left(\mathrm{NH}_{3}\right)_{2}\right]^{+}$, resulting in the formation of a micron-sized silver shell on the surface of the $4 \AA$ molecular sieves. The micronsized hollow silver spheres were obtained by removing the molecular sieve template. SEM and XRD were used to characterize the structure of the micron-sized hollow silver spheres. The as-prepared micro-silver spheres exhibited robust SERS activity in the presence of adsorbed 4-mercaptobenzoic acid (4-MBA) with excitation at $632.8 \mathrm{~nm}$, and the enhancement factor reached $\sim 1.5 \times$ $10^{6}$. This synthetic process represents a promising method for preparing various hollow metal nanoparticles.
\end{abstract}

\section{Introduction}

Surface-enhanced Raman scattering (SERS) is widely used in chemistry, biology, physics, and material science because it can markedly enhance the inherently low Raman scattering signal from molecules leaving the surface of metallic nanoparticles, even at the single-molecule level [1-5]. Recently, photochemical reactions promoted by the two SERS mechanisms have been studied, which are of considerable interest to the researches concerned with nanomaterials preparation, chemical analysis, single-molecule spectroscopy detection, and so forth [6-13]. Several studies have revealed that surface-catalyzed reactions are strongly dependent on the SERS substrates; therefore, much effort has been focused on synthesizing the substrates while maintaining stability, reproducibility, large size reliability, high sensitivity, and low cost [14]. For example, Yamamoto et al. have presented new galvanic displacement reaction, which produced a high SERS intensity substrate [15].
It is known that metal nanoparticles are prone to congregate randomly due to the Van der Waals forces and their high surface energy. Moreover, this aggregation of nanoparticles is difficult to control and results in reducing the stability of SERS substrates. Though some work has been done in the single Ag-NP dimmer to evaluate the plasmon resonance [16], the evaluation is still difficult for the present SERS substrates. In order to prevent the formation of aggregates, metal nanoparticles are often prepared in the presence of stabilizing polymers or various surfactants [1725]. However, these polymers or surfactants interact with the metal substrates and cover their surface, inhibiting the accessibility of the metal nanoparticle surfaces, or even resulting in poor information being collected in experiments. On the other hand, larger noble metal substrates, which are on the submicrometer or micrometer scale, have been found to be capable of reducing aggregation and to provide stable SERS signals for the molecules being investigated [26-29]. In general, once the size of the micron-sized metal spheres 
is close to or higher than the excitation wavelength, their surface enhanced effect is attenuated due to the appearance of the higher-order multipolar plasmon modes. On the other hand, strong electromagnetic field enhancement is provided by the introduction of controllable nanoscale roughness on the particle surface, which can effectively couple to the optical electric field [28].

In our previous studies, micron-sized hollow copper spheres were shown to be effective SERS substrates for characterization of molecular adsorbates. This was mainly attributed to their novel structure with larger specific surface areas [30]. To date, silver has been a reasonable firstclass enhancing SERS substrate because of its narrow and pronounced plasmon resonance [3]. However, the standard electrode potential of the $\left[\mathrm{Ag}\left(\mathrm{NH}_{3}\right)_{2}\right]^{+} / \mathrm{Ag}$ pair is higher than that of the $[\mathrm{Cu}(\mathrm{EDTA})]^{2+} / \mathrm{Cu}$ pair, which will result in the higher proportion of a bulk reduction. Thus, the formation of the silver layer mainly originates from simple deposition after a bulk reduction instead of successive surface reduction of silver ions. As a consequence of weak linkage between the silver nanoparticles, the shells break down following the removal of the core. Even though submicron-sized hollow silver spheres could be synthesized by using soft-template methods, polymers and surfactants had to be used for this study [31]. So it is reasonable to conclude that the synthesis of hollow silver spheres should be more difficult than that of copper. Whether micrometer-sized hollow silver spheres could be synthesized as a drastic SERS substrate is still a scientific problem to be resolved.

In this paper, a hard template method was employed to synthesize micron-sized hollow silver spheres. The $4 \AA$ molecular sieves and formaldehyde were used as a removable solid template and weak reductant in the process, respectively. The virtue of this method is that we can avoid the use of nonvolatile surfactants and polymers. Moreover, the cost of using micron-sized hollow silver spheres is much lower than that of micron-sized solid spheres.

\section{Experimental Section}

2.1. Materials. Silver nitrate (99.8\%) was purchased from Junsei Chemical Co. Ltd. (Japan) and used as received. Ammonia solution (28.0-30.0 wt\%) was obtained from Samchun Pure Chemical Co. Ltd., South Korea. Potassium hydroxide (>82\%) was acquired from Regent Chemical Co. Ltd., Tianjin, China. Formaldehyde (36\%) was obtained from Tianjin Institute of Chemicals, China. Ethanol (99.9\%) was purchased from J. T. Baker, made in Malaysia. Hydrofluoric acid (40\%) was supplied by Tianjin Institute of Chemicals, China. 4-MBA was supplied by Tokyo Kasei Kogyo Co., Ltd, and was used as supplied by the vendor. Four angstrom molecular sieves were supplied from the Dalian Institute of Chemical Physics, Chinese Academy of Sciences, and were heated for $4 \mathrm{~h}$ at $600^{\circ} \mathrm{C}$ in a muffle oven and then cooled in a desiccator. Other chemicals, unless otherwise specified, were of reagent grade and were used without further purification. Highly pure water with resistivity higher than $18.0 \mathrm{M} \Omega \mathrm{cm}$ was used in all the preparations.
2.2. Preparation of the Micron-Sized Hollow Silver Spheres. Molecular sieves coated with silver particles were prepared using a modificatory method described by Saito and coworkers [32]. Here, a potassium hydroxide aqueous solution $(2.6 \mathrm{wt} \%, 0.2 \mathrm{~mL})$ was mixed with a silver nitrate aqueous solution $(2 \mathrm{wt} \%, 3 \mathrm{~mL})$ to form a fine brown precipitate of $\mathrm{Ag}_{2} \mathrm{O}$. An aqueous ammonia solution (8 $\mathrm{wt} \%$ ) was added to the above mixture drop by drop until the precipitate completely dissolved to form $\left[\mathrm{Ag}\left(\mathrm{NH}_{3}\right)_{2}\right]^{+}$. Then a silver nitrate aqueous solution was added to the mixture until the solution became pale brown/yellow. When one drop of ammonia solution ( $2 \mathrm{wt} \%$ ) caused the solution to become transparent again, the addition of ammonia solution to the mixture was stopped. Subsequently, $70 \mathrm{mg}$ of $4 \AA$ molecular sieves was put into the above $\left[\mathrm{Ag}\left(\mathrm{NH}_{3}\right)_{2}\right]^{+}$solution with continuous magnetic stirring and left there for $5 \mathrm{~min}$ to make the molecular sieves disperse uniformly over the system. Then, formaldehyde aqueous solution $(1 \mathrm{~mL}, 2 \mathrm{wt} \%)$ and pure ethanol $(0.5 \mathrm{~mL})$ were added to this mixture. This mixture was kept at $40^{\circ} \mathrm{C}$ in a water bath for $1 \mathrm{~h}$. The reactive solid particles were finally separated from the reaction medium by centrifuging at $3000 \mathrm{rpm}$. After thoroughly washing with water and ethanol, the powdered products were dried under vacuum at room temperature for $4 \mathrm{~h}$.

Due to the permeation of the acid, subsequent treatment with dilute hydrofluoric acid could dissolve the molecular sieve cores to obtain hollow silver spheres. Specifically, $30 \mathrm{mg}$ of Ag-coated $4 \AA$ molecular sieves was initially poured into $50 \mathrm{~mL}$ of $7 \mathrm{wt} \%$ aqueous HF solution and then mixed by hand using the shaking motion at a frequency of $10 \mathrm{~min}^{-1}$. After standing for $1 \mathrm{~h}$, the hollow silver spheres were then separated from the reaction medium by centrifuging at $3000 \mathrm{rpm}$. After thoroughly washing with water and ethanol to remove the excess reactants, the products were dried under vacuum at room temperature for $4 \mathrm{~h}$. In order to adsorb $4-\mathrm{MBA}$ on the hollow silver spheres, the samples were immersed in an ethanolic solution of 4-MBA $\left(18 \mathrm{~mL}, 1 \mathrm{mmol} \mathrm{L}^{-1}\right)$ for $1 \mathrm{~h}$. After thoroughly washing with water and ethanol to remove excess reactants, the products were dried under vacuum at room temperature for $4 \mathrm{~h}$.

\subsection{Characterization of As-Synthesized Micron-Sized Hollow} Silver Spheres. Various methods were used to systematically analyze the composition, structure, morphology, and optical property of the as-synthesized hollow silver spheres. Field emission scanning electron microscope (FESEM) images of samples were recorded with a SUPRA35 FESEM operating at $15.00 \mathrm{kV}$. Mixed $4 \AA$ molecular sieves into $\left[\mathrm{Ag}\left(\mathrm{NH}_{3}\right)_{2}\right]^{+}$solution, then formaldehyde aqueous solution $(2 \mathrm{wt} \%)$ and pure ethanol were added into this mixture, water bath for $1 \mathrm{~h}$. The size distribution of the $4 \AA$ molecular sieves was calculated with LS-100Q laser diffraction particle size analyzer. X-ray powder diffraction (XRD) measurements were performed on a D8ADVANCE powder diffractometer over a $2 \theta$ range of $5-90^{\circ}$ at an angular resolution of $0.04^{\circ}$. Raman spectra were obtained with a Jobin Yvon LabRam HR 800 micro-Raman spectrometer equipped with a microscope (Panasonic Super DYNAMIC). $632.8 \mathrm{~nm}$ laser radiation was used for excitation. 


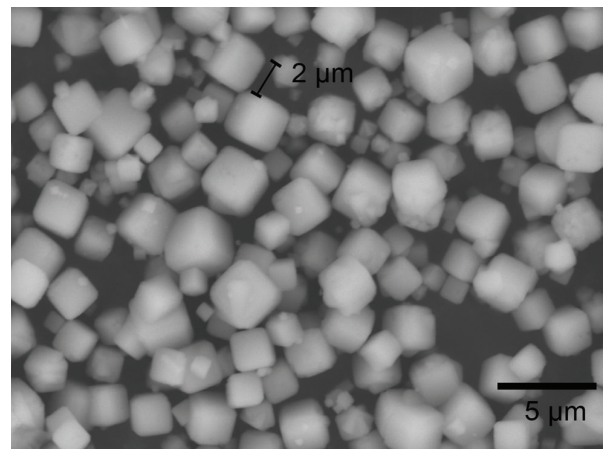

(a)

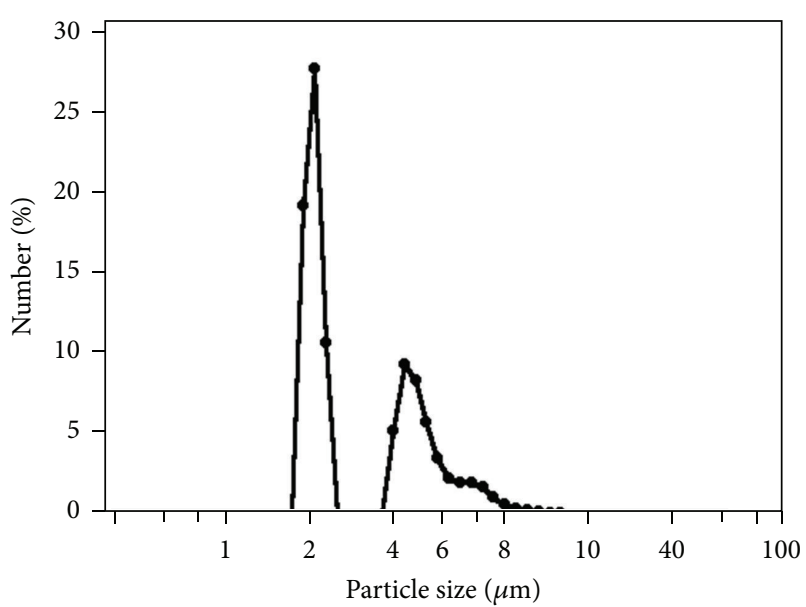

(b)

FIGURE 1: (a) FESEM image of 4 Å molecular sieves and (b) distribution of particle sizes.

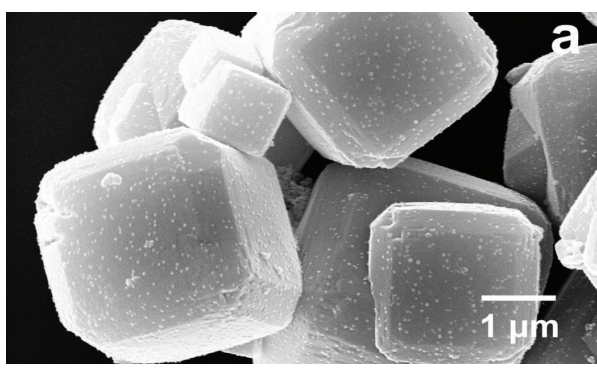

(a)

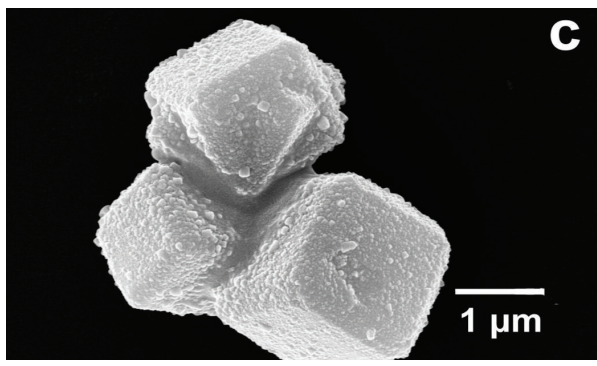

(c)

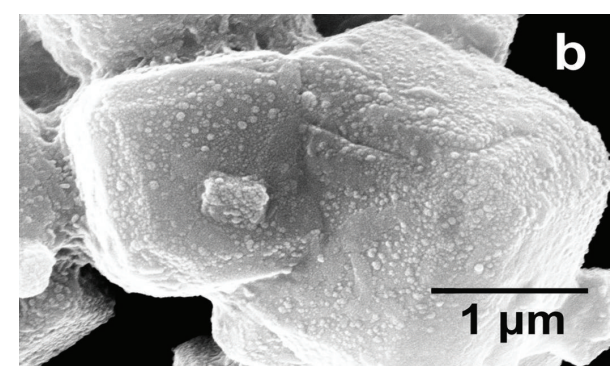

(b)

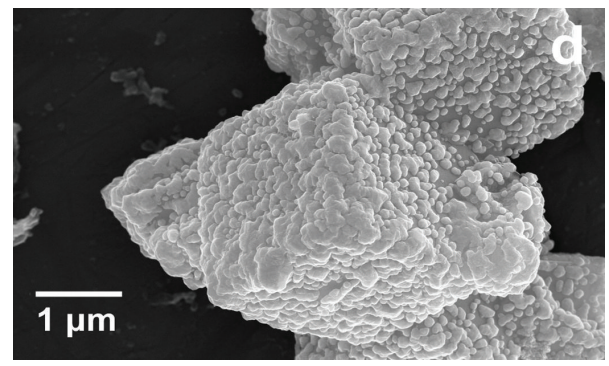

(d)

FIGURE 2: FESEM image of the formation process of Ag-coated molecular sieves.

The Raman band of a silicon wafer at $520 \mathrm{~cm}^{-1}$ was used to calibrate the spectrometer.

\section{Results and Discussion}

3.1. Design and Surface Analysis of Reproducible SERS Substrate. The surface completeness, morphology, and size distribution of the uncoated and Ag-coated $4 \AA$ molecular sieves as well as the hollow silver spheres were analyzed by FESEM. As can be seen from Figure 1(a), the typical particles of the molecular sieves were cuboidal in shape, with their sizes ranging from 1 to $2 \mu \mathrm{m}$. Although the surfaces of these molecular sieves look intact and slick, actually, there are many
$4 \AA$ holes on the surfaces. Further, the laser diffraction data indicates that most of the particles are $2 \mu \mathrm{m}$ in size (see Figure 1(b)), which is in good agreement with the FESEM results.

Figures 2(a)-2(d) present the formation process of Agcoated molecular sieves. Clearly, the $\left[\mathrm{Ag}\left(\mathrm{NH}_{3}\right)_{2}\right]^{+}$ions that were adsorbed on the surface of the molecular sieves were slowly reduced, and then they acted as seeds for electroless plating of silver, from which the interconnected $\mathrm{Ag}$ particles on the external surface of the $4 \AA$ molecular sieves were formed. Finally, a relatively continuous silver layer was obtained. As can be seen from the FESEM image of the Agcoated molecular sieves and as shown in Figure 2(d), the sizes of the coated particles were $1-3 \mu \mathrm{m}$ and were densely 


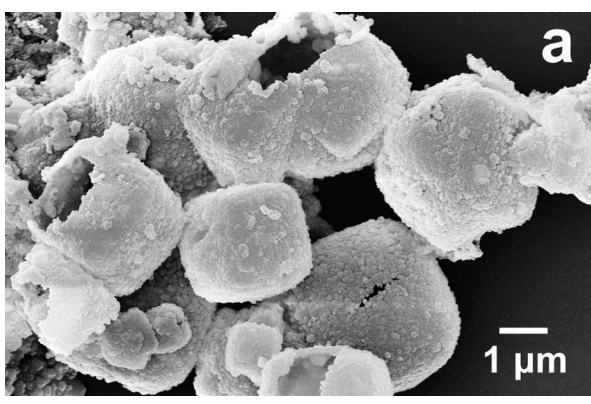

(a)

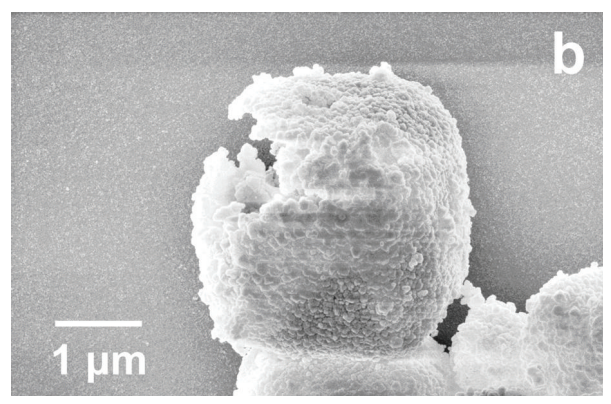

(b)

FIGURE 3: FESEM images of (a) the hollow silver spheres and (b) the hollow silver spheres at higher magnification.

distributed with silver nanoparticles that were several tens of nanometers in size. Following the dissolution of the core, hollow silver spheres were obtained. As can be seen from Figure 3 , they are still approximately cuboidal in shape, with their particle sizes ranging from 1 to $3 \mu \mathrm{m}$. In addition, their surfaces were quite rough, which is believed to be of benefit for their applications in SERS-based devices. So we concluded that the micron-sized hollow silver spheres can be readily synthesized by using $4 \AA$ molecular sieves and formaldehyde as the removable solid template and weak reductant, respectively. To the best of our knowledge, this is the first time that hollow silver spheres have been synthesized by using low-cost and easy-removal molecular sieves as a solid template.

As is well known, the formation of good hollow silver spheres is correlated with the intrinsic nature of the template and reductant used. Firstly, $\left[\mathrm{Ag}\left(\mathrm{NH}_{3}\right)_{2}\right]^{+}$ions can be easily adsorbed and reduced on the surface of $4 \AA$ molecular sieves due to their negatively charged oxygen sites. Based on this, the surface reduction of silver ions, instead of simple deposition after a bulk reduction, will result in the formation of a silver layer. Also the shell structure after removal of the core can be retained. Secondly, our previous studies have revealed that if a strong reducing agent (such as glucose) was used, the silver shells will be broken and the nanostructures will aggregate once the cores are removed. This is due to the weak link between the silver nanoparticles because the particles in this study originated from the simple deposition after a bulk reduction instead of successive surface reduction of silver ions [33]. This motivated us to select formaldehyde as the reducing agent in the electroless silver-plating process. Moreover, ammonia is used as a complexing agent to hold most of the silver ions in solution and prevent random deposition. In this way, a good silver layer can be obtained because of the relatively lower difference in the redox potentials between the reducing agent and the metal.

In order to confirm the composition of the hollow silver spheres, XRD measurements were performed on the D8ADVANCE powder diffractometer over a $2 \theta$ range of 5$90^{\circ}$ at an angular resolution of $0.04^{\circ}$. Figure 4 shows typical XRD patterns of the uncoated and Ag-coated $4 \AA$ molecular sieves as well as the hollow silver spheres. Comparing line $\mathrm{B}$ with line $\mathrm{A}$ in Figure 4, it is very clear that several new peaks appeared. For instance, the $2 \theta$ values of $38.12^{\circ}, 44.38^{\circ}$,

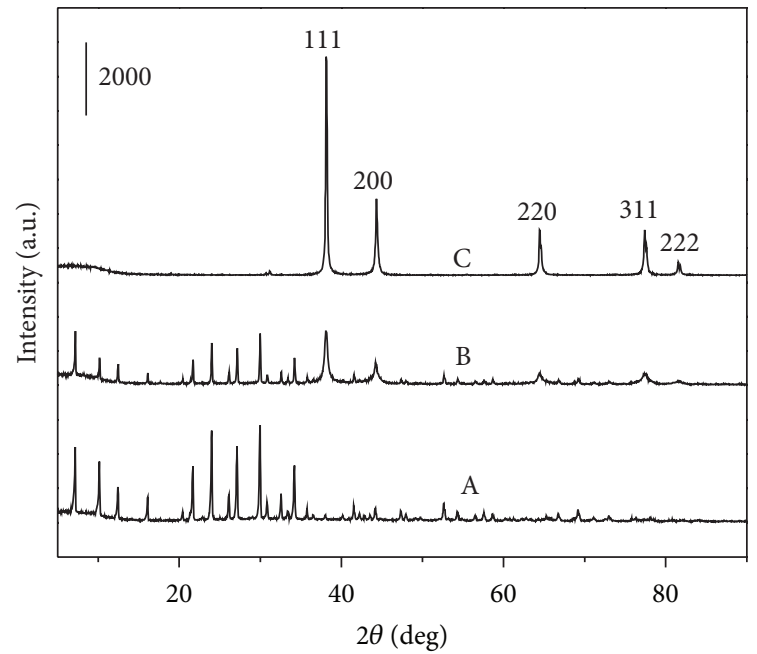

FIgURE 4: XRD patterns of 4 Å molecular sieves (line A), Ag-coated molecular sieves (line B), and hollow silver spheres (line C).

$64.38^{\circ}, 77.46^{\circ}$, and $81.52^{\circ}$ correspond to the reflections of the $\{111\},\{200\},\{220\},\{311\}$, and $\{222\}$ crystal planes of the metallic Ag with cubic structure, respectively. They agree well with the standard values given by the Joint Committee on Powder Diffraction Standards. So the fact that the surfaces of the $4 \AA$ molecular sieves were coated by crystallized $\mathrm{Ag}$ can be confirmed. Furthermore, as can be seen from the phase analysis of the hollow silver spheres by XRD (line C in Figure 4), only silver diffraction peaks were observed, which indicates that pure hollow silver spheres were obtained after the removal of the molecular sieve cores by dilute hydrofluoric acid.

3.2. SERS Activity of the Reproducible SERS Substrate. The SERS activity of the hollow silver spheres was evaluated by using 4-MBA as the probing adsorbate with excitation at $632.8 \mathrm{~nm}$. Figure 5 shows the Raman scattering spectra obtained from the dry pure 4-MBA powder (line A) and from 4-MBA adsorbed on the hollow silver spheres (line B), which were normalized with respect to the absolute intensity from a silicon wafer. Clearly, significant differences in peak positions and their relative intensities between these two 


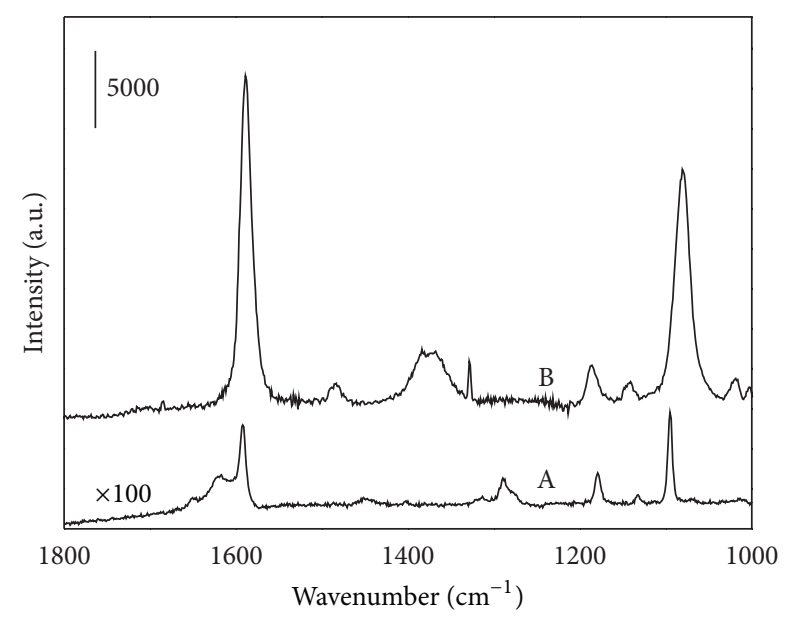

Figure 5: Raman spectra of 4-MBA adsorbed on the hollow silver spheres (line B) and dry 4-MBA powder (line A).

Raman spectra can be observed. All the peaks in line B in Figure 5 can be attributed to the Raman signals of the 4MBA adsorbed on the silver substrates. According to previous studies, the strong Raman peak at about $1589 \mathrm{~cm}^{-1}$ arose from $v_{8 a}$ aromatic-ring vibrations, and the Raman band at about $1081 \mathrm{~cm}^{-1}$ was due to $v_{12}$ aromatic-ring vibrations possessing C-S stretching characteristics; additionally, other bands could also be assigned to proper ring modes [34]. The SERS enhancement factors (EF) can be estimated by using the following equation:

$$
\mathrm{EF}=\frac{\left(I_{\mathrm{SERS}} / N_{\mathrm{SERS}}\right)}{\left(I_{\mathrm{NR}} / N_{\mathrm{NR}}\right)},
$$

where $I_{\text {SERS }}$ denotes the SERS intensity of the $v_{8 a}$ band of 4MBA adsorbed on the hollow silver spheres, $I_{\mathrm{NR}}$ represents the normal Raman (NR) scattering intensity of the same band of dry 4-MBA powder, and $N_{\mathrm{SERS}}$ and $N_{\mathrm{NR}}$ represent the numbers of 4-MBA molecules effectively excited by the laser beam to obtain the corresponding SERS and NR spectra, respectively. First of all, we assumed that the sampling volume is the product of the laser spot $(\sim 2 \mu \mathrm{m}$ in diameter) and the penetration depth $(\sim 2 \mu \mathrm{m})$ of the focused beam, from which the NR spectrum of dry 4-MBA powder was obtained (as shown by line A in Figure 5). Since the density of 4-MBA is $1.5 \mathrm{~g} \cdot \mathrm{cm}^{-3}$ and its molar mass is $154.19 \mathrm{~g} \cdot \mathrm{mol}^{-1}$, the number of 4-MBA molecules effectively excited by the laser beam was calculated to be $3.68 \times 10^{10}$ (i.e., $6.11 \times 10^{-14} \mathrm{~mol}$ ). Secondly, the bonding density of 4-MBA molecules in a self-assembled monolayer is $\sim 0.5 \mathrm{nmol} \cdot \mathrm{cm}^{-2}$ [35]. Consequently, the total number of molecules sampled in the SERS experiments should be $9.45 \times 10^{6}$ (i.e., $1.57 \times 10^{-17} \mathrm{~mol}$ ). Please note that we ignore the surface roughness of the samples in this estimation. Based on the above analysis, the intensity ratio of the $v_{8 a}$ aromatic-ring vibration modes in Figure 5 is $382: 1$, and the EF of the hollow silver spheres, estimated by using 4 -MBA as the probing adsorbate, reaches $\sim 1.5 \times$ $10^{6}$. This indicates that the hollow silver spheres can be used as a better performance SERS substrate. We recall the fact that the influence of the substrate micromorphology on optical properties is significant [36]. Thus, the most likely explanation for the better SERS enhancement of the hollow silver spheres is that their rough surfaces result in greater localized field enhancement.

Finally, we have to emphasize that the valuable advantage of our synthesis method is that no polymers or surfactants are involved in the process, which are believed to bring about spurious peaks and to hinder the use of SERS-active substrates for analytical purposes. In most cases, the addition of polymers or surfactants leads to some decrease in the enhancement effect due to the reduced accessibility of the particle surface, and the adsorption of various molecules may be inhibited due to the predominant adsorption of undesirable polymers or surfactants resulting in inaccurate information being gathered. Despite the fact that several cleaning cycles are needed, Velikov and coworkers have demonstrated that the particles still contained a small amount of protective polymers or surfactants once they were used in particle preparations, and this significantly affected the SERS properties of the metallic nanoparticles [27]. Furthermore, from the application point of view, the hollow silver spheres cost less compared to solid spheres; moreover, hollow spheres have stronger SERS enhancement than that of Ag-coated silica spheres [37]. In addition, the framework of the $4 \AA$ molecular sieves was made up of tetrahedrons of $\mathrm{SiO}_{4}$ and $\mathrm{AlO}_{4}$ and their surfaces consisted entirely in negatively charged oxygen sites. So $\left[\mathrm{Ag}\left(\mathrm{NH}_{3}\right)_{2}\right]^{+}$ions could easily be adsorbed and be reduced at those locations; in addition, the various stages of pretreatment for the core material can be omitted in this process. This is the superiority of the $4 \AA$ molecular sieves, used as templates for constructing a silver layer, when compared with silica particles prepared using the Stöber et al's procedure [38]. In that case, some $\mathrm{Si}-\mathrm{O}-\mathrm{C}_{2} \mathrm{H}_{5}$ bonds remain on the surface of the silica particles owing to the incomplete hydrolysis of the tetraethyl orthosilicate. As a consequence, $\left[\mathrm{Ag}\left(\mathrm{NH}_{3}\right)_{2}\right]^{+}$ions were not adsorbed easily, and the surface charge density becomes very low.

\section{Conclusions}

A new, well-designed type of micron-sized hollow silver sphere has been synthesized and confirmed by FESEM coupled with XRD characterization. It was found that the $4 \AA$ molecular sieves template is an effective template owing to the presence of many active negatively charged oxygen sites and holes on its surface. The strongly enhanced Raman scattering from the monolayer film of the 4-MBA probe molecules with excitation at $632.8 \mathrm{~nm}$ showed that the as-prepared micronsized hollow silver spheres are SERS active, and the EF was calculated to be $\sim 1.5 \times 10^{6}$. In comparison with the micronsized solid metal spheres, three features become apparent. (1) No nonvolatile polymers or surfactants were used in the preparation processes of the hollow silver spheres. (2) This approach is easier to control than the approach for preparing micron-sized solid metal spheres. (3) The surface charge density is largely increased due to the use of the $4 \AA$ molecular sieves template for constructing the silver layer. The new 
hollow silver spheres were produced in a simple and costeffective method. They are expected to play an important role in catalysts, fillers, engineering, and SERS-based analytical devices. The synthetic route may become a promising option for producing hollow metal materials.

\section{Conflict of Interests}

The authors declare that there is no conflict of interests regarding the publication of this paper.

\section{Acknowledgments}

This work was supported by the National Natural Science Foundation of China (21271095), the Doctor Subject Foundation of the Ministry of Education of China (20132101110001), the Liaoning Natural Science Foundation of China (2013020100 and 2013004003), and the Shenyang Natural Science Foundation of China (F13-289-1-00, F12-2654-00, and F14-191-4-00). Peng Song is indebted to the financial support from the Program of Liaoning Key Laboratory of Semiconductor Light Emitting and Photocatalytic Materials.

\section{References}

[1] S. Nie and S. R. Emory, "Probing single molecules and single nanoparticles by surface-enhanced Raman scattering," Science, vol. 275, no. 5303, pp. 1102-1106, 1997.

[2] K. Kneipp, Y. Wang, H. Kneipp et al., "Single molecule detection using surface-enhanced Raman scattering (SERS)," Physical Review Letters, vol. 78, no. 9, pp. 1667-1670, 1997.

[3] M. T. Sun, S. S. Liu, H. X. Chen, and J. Raman, "Direct visual evidence for the chemical mechanism of surface-enhanced resonance Raman scattering via charge transfer," Journal of Raman Spectroscopy, vol. 40, no. 2, pp. 137-143, 2009.

[4] Y. Fang, Y. Li, H. Xu, and M. Sun, "Ascertaining p, p'dimercaptoazobenzene produced from $\mathrm{p}$-aminothiophenol by selective catalytic coupling reaction on silver nanoparticles," Langmuir, vol. 26, no. 11, pp. 7737-7746, 2010.

[5] K. Ueno and H. Misawa, "Surface plasmon-enhanced photochemical reactions," Journal of Photochemistry and Photobiology C: Photochemistry Reviews, vol. 15, no. 1, pp. 31-52, 2013.

[6] H. Xu, E. J. Bjerneld, M. Käll, and L. Börjesson, "Spectroscopy of single hemoglobin molecules by surface enhanced raman scattering," Physical Review Letters, vol. 83, no. 21, pp. 43574360, 1999.

[7] A. M. Michaels, J. Jiang, and L. Brus, "Ag nanocrystal junctions as the site for surface-enhanced raman scattering of single rhodamine 6 g molecules," Journal of Physical Chemistry B, vol. 104, no. 50, pp. 11965-11971, 2000.

[8] Y. Maruyama, M. Ishikawa, and M. Futamata, "Surface-enhanced Raman scattering of single adenine molecules on silver colloidal particles," Chemistry Letters, vol. 30, pp. 834-835, 2001.

[9] M. Futamata, Y. Maruyama, and M. Ishikawa, "Adsorbed sites of individual molecules on Ag nanoparticles in single molecule sensitivity-surface-enhanced Raman scattering," Journal of Physical Chemistry B, vol. 108, no. 35, pp. 13119-13127, 2004.

[10] E. C. Le Ru, M. Meyer, and P. G. Etchegoin, "Proof of singlemolecule sensitivity in Surface Enhanced Raman Scattering
(SERS) by means of a two-analyte technique," Journal of Physical Chemistry B, vol. 110, no. 4, pp. 1944-1948, 2006.

[11] J. A. Dieringer, R. B. Lettan II, K. A. Scheidt, and R. P. van Duyne, "A frequency domain existence proof of singlemolecule surface-enhanced Raman spectroscopy," Journal of the American Chemical Society, vol. 129, no. 51, pp. 16249-16256, 2007.

[12] Y. Fang, H. Wei, F. Hao, P. Nordlander, and H. Xu, "Remoteexcitation surface-enhanced raman scattering using propagating ag nanowire plasmons," Nano Letters, vol. 9, no. 5, pp. 20492053, 2009.

[13] S. L. Kleinman, E. Ringe, N. Valley et al., "Single-molecule surface-enhanced raman spectroscopy of crystal violet isotopologues: Theory and experiment," Journal of the American Chemical Society, vol. 133, no. 11, pp. 4115-4122, 2011.

[14] M. Sun and H. Xu, "A novel application of plasmonics: plasmondriven surface-catalyzed reactions," Small, vol. 8, no. 18, pp. 2777-2786, 2012.

[15] Y. S. Yamamoto, K. Hasegawa, Y. Hasegawa et al., "Direct conversion of silver complexes to nanoscale hexagonal columns on a copper alloy for plasmonic applications," Physical Chemistry Chemical Physics, vol. 15, no. 35, pp. 14611-14615, 2013.

[16] Y. S. Yamamoto, M. Ishikawa, Y. Ozaki, and T. Itoh, "Fundamental studies on enhancement and blinking mechanism of surfaceenhanced Raman scattering (SERS) and basic applications of SERS biological sensing," Frontiers of Physics, vol. 9, no. 1, pp. 31-46, 2014.

[17] H. Lee, S. E. Habas, S. Kweskin, D. Butcher, G. A. Somorjai, and P. Yang, "Morphological control of catalytically active platinum nanocrystals," Angewandte Chemie-International Edition, vol. 45, no. 46, pp. 7824-7828, 2006.

[18] T. S. Ahmadi, Z. L. Wang, T. C. Green, A. Henglein, and M. A. El-Sayed, "Shape-controlled synthesis of colloidal platinum nanoparticles," Science, vol. 272, no. 5270, pp. 1924-1925, 1996.

[19] T. Pal, T. K. Sau, and N. R. Jana, "Reversible formation and dissolution of silver nanoparticles in aqueous surfactant media," Langmuir, vol. 13, no. 6, pp. 1481-1485, 1997.

[20] T. Pal, T. K. Sau, and N. R. Jana, "Silver hydrosol, organosol, and reverse micelle-stabilized sol-a comparative study," Journal of Colloid and Interface Science, vol. 202, no. 1, pp. 30-36, 1998.

[21] J. H. Fendler, "Atomic and molecular clusters in membrane mimetic chemistry," Chemical Reviews, vol. 87, no. 5, pp. 877899, 1987.

[22] G. Schmid, "Large clusters and colloids. Metals in the embryonic state," Chemical Reviews, vol. 92, no. 8, pp. 1709-1727, 1992.

[23] L. N. Lewis, "Chemical catalysis by colloids and clusters," Chemical Reviews, vol. 93, no. 8, pp. 2693-2730, 1993.

[24] L. Longenberger and G. Mills, "Formation of metal particles in aqueous solutions by reactions of metal complexes with polymers," Journal of Physical Chemistry, vol. 99, no. 2, pp. 475478, 1995.

[25] W. Tu, K. Takai, K. I. Fukui, A. Miyazaki, and T. Enoki, "Interface effect on the electronic structure of alkanethiol-coated platinum nanoparticles," Journal of Physical Chemistry B, vol. 107, no. 37, pp. 10134-10140, 2003.

[26] D. V. Goia and E. Matijević, "Preparation of monodispersed metal particles," New Journal of Chemistry, vol. 22, no. 11, pp. 1203-1215, 1998.

[27] K. P. Velikov, G. E. Zegers, and A. van Blaaderen, "Synthesis and characterization of large colloidal silver particles," Langmuir, vol. 19, no. 4, pp. 1384-1389, 2003. 
[28] H. Wang and N. J. Halas, "Mesoscopic au "meatball" particles," Advanced Materials, vol. 20, no. 4, pp. 820-825, 2008.

[29] H. Liang, Z. Li, W. Wang, Y. Wu, and H. Xu, "Highly surfaceroughened "flower-like" silver nanoparticles for extremely sensitive substrates of surface-enhanced raman scattering," Advanced Materials, vol. 21, no. 45, pp. 4614-4618, 2009.

[30] L. Xia, G. Liu, J. Wang, and S. Luo, "Facile synthesis of micronsized hollow copper spheres with ZSM-5 molecular sieve as a template," Journal of Raman Spectroscopy, vol. 40, no. 8, pp. 876880, 2009.

[31] D. Zhang, L. Qi, J. Ma, and H. Cheng, "Synthesis of submicrometer-sized hollow silver spheres in mixed polymersurfactant solutions," Advanced Materials, vol. 14, no. 20, pp. 1499-1502, 2002.

[32] Y. Saito, J. J. Wang, D. A. Smith, and D. N. Batchelder, "A simple chemical method for the preparation of silver surfaces for efficient SERS," Langmuir, vol. 18, no. 8, pp. 2959-2961, 2002.

[33] L. Xia, J. Wang, S. Tong, G. Liu, J. Li, and H. Zhang, "Design and construction of a sensitive silver substrate for surface-enhanced Raman scattering spectroscopy," Vibrational Spectroscopy, vol. 47, no. 2, pp. 124-128, 2008.

[34] A. Michota and J. Bukowska, "Surface-enhanced Raman scattering (SERS) of 4-mercaptobenzoic acid on silver and gold substrates," Journal of Raman Spectroscopy, vol. 34, no. 1, pp. 21$25,2003$.

[35] C. J. Orendorff, A. Gole, T. K. Sau, and C. J. Murphy, "Surfaceenhanced Raman spectroscopy of self-assembled monolayers: sandwich architecture and nanoparticle shape dependence," Analytical Chemistry, vol. 77, no. 10, pp. 3261-3266, 2005.

[36] B. Rodríguez-Gonzélez, V. Salgueiriño-Maceira, F. GarcíaSantamaría, and L. M. Liz-Marzán, "Fully accessible gold nanoparticles within ordered macroporous solids," Nano Letters, vol. 2, no. 5, pp. 471-473, 2002.

[37] K. Kim, H. S. Kim, and H. K. Park, "Facile method to prepare surface-enhanced-Raman-scattering-active Ag nanostructures on silica spheres," Langmuir, vol. 22, no. 19, pp. 8083-8088, 2006.

[38] W. Stöber, A. Fink, and E. Bohn, "Controlled growth of monodisperse silica spheres in the micron size range," Journal of Colloid And Interface Science, vol. 26, no. 1, pp. 62-69, 1968. 

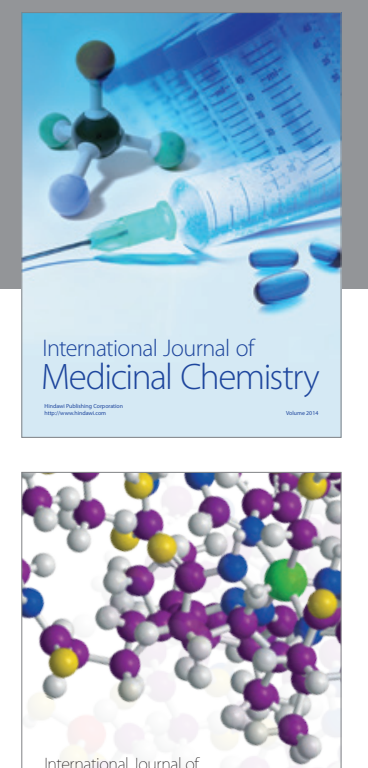

\section{Carbohydrate} Chemistry

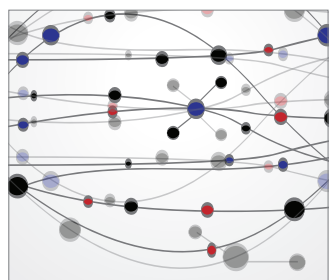

The Scientific World Journal
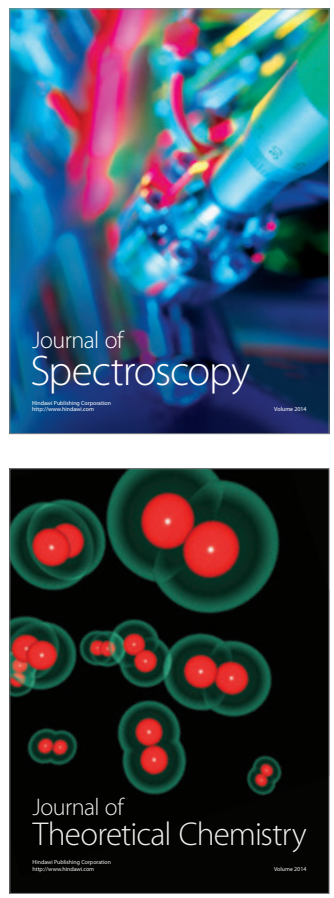
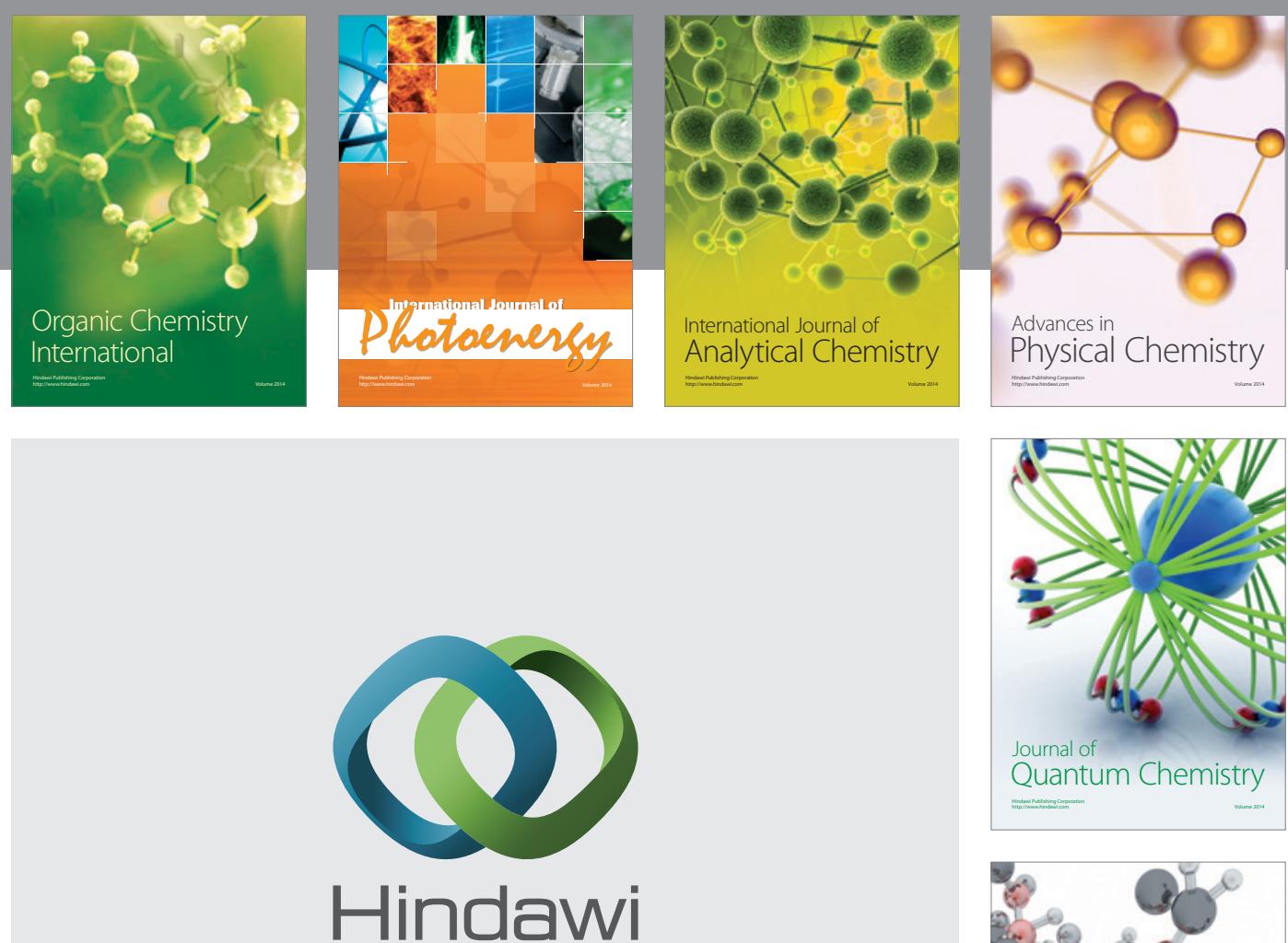

Submit your manuscripts at

http://www.hindawi.com

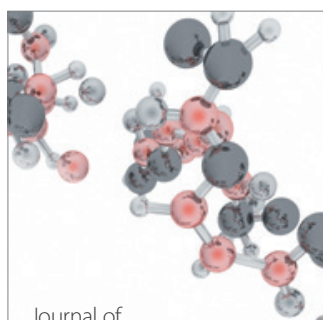

Analytical Methods

in Chemistry

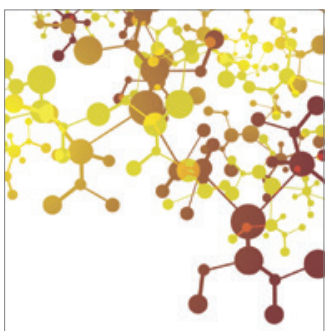

Journal of

Applied Chemistry

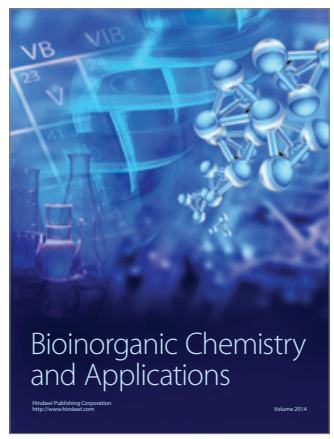

Inorganic Chemistry
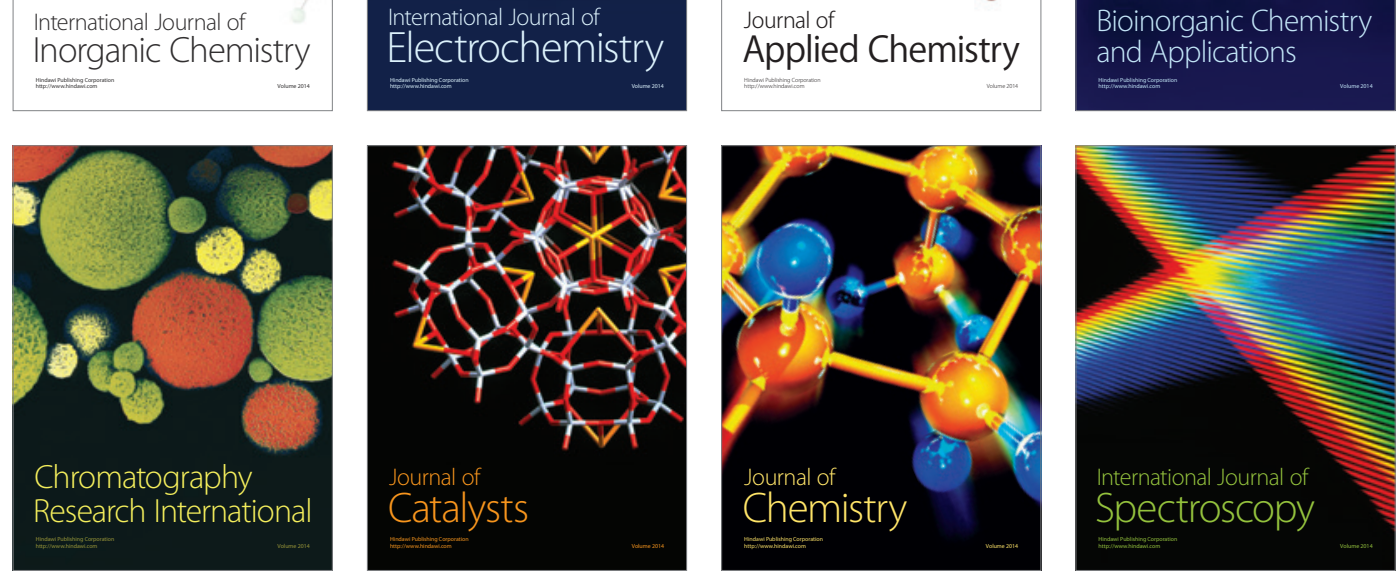